\title{
ISOTERMA DE ADSORÇÃO DE UMIDADE DA CASTANHA-DO-BRASIL (Bertholletia excelsa)
}

\section{MOISTURE ADSORPTION ISOTHERM FROM BRAZIL-NUT (Bertholletia excelsa)}

\author{
Renan Campos Chisté ${ }^{1}$; Alessandra Santos Lopes ${ }^{2}$; Rosinelson da Silva Pena ${ }^{2}$ \\ ${ }^{1}$ Universidade Estadual de Campinas - UNICAMP - Campinas - Brasil - renanchiste@gmail.com \\ ${ }^{2}$ Universidade Federal do Pará - UFPA - Belém - Brasil
}

\begin{abstract}
Resumo
Realizou-se o estudo do comportamento higroscópico da castanha-do-Brasil construindo a isoterma de adsorção de umidade a $25{ }^{\circ} \mathrm{C}$, na faixa de atividade de água $\left(a_{w}\right)$ de 0,29 a 0,96. Avaliou-se a aplicabilidade de oito modelos matemáticos (Kuhn, Handerson, Oswin, Halsey, Smith, GAB, BET e BET-modificada) na predição da isoterma. O produto apresentou isoterma do Tipo II e foi observado o desenvolvimento de fungos em $a_{w}$ superior a 0,85. Para assegurar estabilidade microbiológica $\left(a_{w}<0,6\right)$ a umidade do produto não deve ser superior a 1,62 g $\mathrm{H}_{2} \mathrm{O} / 100 \mathrm{~g}$ b.s. $\mathrm{O}$ valor da monocamada de 0,7 $\mathrm{g} \mathrm{H}_{2} \mathrm{O} / 100 \mathrm{~g}$ b.s. indicou a quase inexistência de constituintes com afinidade por água no produto. As equações de Kuhn, Halsey, GAB, BET e BET-modificada foram capazes de predizer a isoterma de adsorção do produto, sendo mais indicada a equação de Kuhn, por ser bi-paramétrica e por ter apresentado o melhor ajuste aos pontos experimentais.
\end{abstract}

Palavras-chave: modelos matemáticos; higroscopicidade; atividade de água.

\section{Introdução}

A castanheira-do-Brasil (Bertholletia excelsa, H.B.K.) é originária da região Amazônica. Suas amêndoas são oleaginosas, com elevado valor energético, e ricas em proteínas. Apresenta também constituintes importantes como o selênio, que vem sendo referido na prevenção de câncer e doenças cardiovasculares. O consumo de suas amêndoas no mercado interno é pequeno e estimado em $1 \%$ da produção. A maior parte é exportada in natura para países da Europa (Alemanha e Inglaterra) e da América do Norte (Estados Unidos) (Glória; Regitano-D’arce, 2000; Souza; Menezes, 2004). No Brasil, a produção total de castanha-do-Brasil em 2009 atingiu cerca de 37,5 mil toneladas/ano, sendo a região norte a maior produtora (35 mil toneladas/ano), representando aproximadamente $93 \%$ da produção nacional, seguida da região centro-oeste $(1,5$ mil toneladas/ano) (IBGE, 2010). 
A estabilidade física, química e microbiológica dos alimentos é dada principalmente pela relação entre o teor da umidade de equilíbrio da matéria alimentícia e sua atividade de água $\left(\mathrm{a}_{\mathrm{w}}\right)$, em uma dada temperatura (Myara et al., 1998). As isotermas de sorção são únicas para cada tipo de alimento e podem ser empregadas diretamente na resolução de problemas, no processamento de alimentos, predições da energia necessária ao processo e determinação das condições de armazenamento.

Equações (ou modelos matemáticos) para isotermas de sorção de água podem ser utilizadas na predição da adsorção ou dessorção de umidade em matérias alimentícias. Muitas equações empíricas e semi-empíricas descrevendo as características de sorção de alimentos têm sido propostas na literatura. Labuza (1975) atribui tais utilizações ao fato de que a água é associada à matriz alimentícia por diferentes mecanismos, em diferentes regiões de atividade de água.

Através das isotermas pode-se determinar a monocamada, a qual além de estar relacionada com o início de uma série de reações químicas de deterioração dos alimentos, representa o ponto de maior gasto de energia em um processo de secagem (Assunção e Pena, 2007).

A castanha-do-Brasil é estocada por longos períodos, seja para consumo no mercado interno brasileiro ou durante a exportação para o mercado externo. Portanto, o objetivo deste trabalho visa o estudo do comportamento higroscópico, assim como a aplicabilidade de oito modelos matemáticos na predição dos dados de adsorção de umidade da Castanha-do-Brasil.

\section{Material e Métodos}

\section{Matéria-prima}

As amêndoas de castanha-do-Brasil utilizadas no estudo foram fornecidas por uma empresa exportadora, localizada no município de Belém, Pará, Brasil. As amostras foram trituradas em processador de alimentos, homogeneizadas, e imediatamente utilizadas na obtenção dos dados de adsorção de umidade.

\section{Obtenção dos dados de adsorção de umidade}

Na obtenção dos dados de adsorção, amostras das amêndoas trituradas, devidamente pesadas $(\approx 1 \mathrm{~g})$, em recipientes do aparelho de atividade de água, foram submetidas à desidratação completa, em dessecador contendo sílica-gel na base, sob vácuo, à temperatura ambiente $\left(\approx 25^{\circ} \mathrm{C}\right)$, por um período de 24 horas. Em seguida, as amostras foram colocadas em dessecador contendo água na base e mantidas na temperatura de trabalho $\left(25^{\circ} \mathrm{C}\right)$, com variação de $\pm 1{ }^{\circ} \mathrm{C}$. 
Durante o tempo de contato retiraram-se amostras em duplicata, em tempos crescentes (equilíbrio dinâmico), para determinar a umidade por diferença de massa, com o auxílio de uma balança analítica e a $a_{w}$ com auxílio de um higrômetro AQUAlab 3TE. Durante todo o ensaio experimental, as amostras foram submetidas à inspeção visual, com a finalidade de observar alterações visualmente perceptíveis, como: caking, escurecimento e crescimento de fungos.

\section{Isoterma de adsorção de umidade}

A isoterma de adsorção foi construída na temperatura de $25^{\circ} \mathrm{C}$, a partir da relação existente entre as umidades do produto e as $\mathrm{a}_{\mathrm{w}}$ correspondentes, com auxílio do aplicativo Microsoft Office Excel 2003 (2002).

\section{Predição da isoterma de adsorção}

A predição dos dados de adsorção de umidade da castanha-do-Brasil foi testada através da utilização de oito modelos matemáticos, cinco bi-paramétricos e três tri-paramétricos (Tabela 1).

Tabela 1 - Modelos utilizados na predição da isoterma de adsorção da castanha-do-Brasil.

\begin{tabular}{|c|c|c|}
\hline & Equação & Modelos \\
\hline \multirow{5}{*}{ 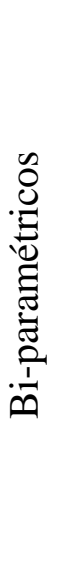 } & $\mathrm{Kuhn}^{\mathrm{a}}$ & $\mathrm{m}=-\frac{\mathrm{a}}{\ln \mathrm{a}_{\mathrm{w}}}+\mathrm{b}$ \\
\hline & Handerson $^{\mathrm{a}}$ & $\mathrm{m}=\left[\frac{-\ln \left(1-\mathrm{a}_{\mathrm{w}}\right)}{\mathrm{a}}\right]^{\frac{1}{\mathrm{~b}}}$ \\
\hline & Oswin $^{\mathrm{a}}$ & $\mathrm{m}=\mathrm{a}\left[\frac{\mathrm{a}_{\mathrm{w}}}{1-\mathrm{a}_{\mathrm{w}}}\right]$ \\
\hline & Halsey $^{\mathrm{a}}$ & $\mathrm{m}=\left[\frac{-\mathrm{a}}{\ln \mathrm{a}_{\mathrm{w}}}\right]^{\mathrm{b}}$ \\
\hline & $\mathrm{Smith}^{\mathrm{a}}$ & $\mathrm{m}=\mathrm{a}-\mathrm{b} \cdot \ln \left(1-\mathrm{a}_{\mathrm{w}}\right)$ \\
\hline \multirow{3}{*}{ 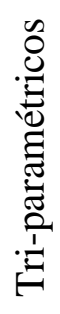 } & $\mathrm{GAB}^{\mathrm{b}}$ & $\mathrm{m}=\frac{\mathrm{m}_{\mathrm{o}} \cdot \mathrm{c} \cdot \mathrm{k} \cdot \mathrm{a}_{\mathrm{w}}}{\left[\left(1-\mathrm{k} \cdot \mathrm{a}_{\mathrm{w}}\right) \cdot\left(1+(\mathrm{c}-1) \cdot \mathrm{k} \cdot \mathrm{a}_{\mathrm{w}}\right)\right]}$ \\
\hline & $\mathrm{BET}^{\mathrm{d}}$ & $\mathrm{m}=\frac{\mathrm{m}_{\mathrm{o}} \cdot \mathrm{c} \cdot \mathrm{a}_{\mathrm{w}}}{1-\mathrm{a}_{\mathrm{w}}} \cdot\left(\frac{1-(\mathrm{n}+1) \cdot \mathrm{a}_{\mathrm{w}}^{\mathrm{n}}+\mathrm{n} \cdot \mathrm{a}_{\mathrm{w}}^{\mathrm{n}+1}}{1-(1-\mathrm{c}) \cdot \mathrm{a}_{\mathrm{w}}-\mathrm{c} \cdot \mathrm{a}_{\mathrm{w}}^{\mathrm{n}+1}}\right)$ \\
\hline & BET modificada ${ }^{c}$ & $\mathrm{~m}=\frac{\mathrm{m}_{\mathrm{o}} \cdot \mathrm{c} \cdot \mathrm{a}_{\mathrm{w}}}{\left[\left(1-\mathrm{k} \cdot \mathrm{a}_{\mathrm{w}}\right) \cdot\left(1+(\mathrm{c}-\mathrm{k}) \cdot \mathrm{a}_{\mathrm{w}}\right)\right]}$ \\
\hline
\end{tabular}


A análise de regressão foi realizada com auxílio do aplicativo STATISTICA for Windows 5.5 (2000), utilizando a metodologia de estimativa Levenberg-Marquardt e critério de convergência de $10^{-6}$. Os parâmetros utilizados para avaliar os ajustes foram: o coeficiente de determinação $\left(\mathrm{R}^{2}\right)$, o desvio médio relativo (P) (Equação 1) e a correlação entre os valores de umidade experimentais e preditos. Valores de P menores que $10 \%$ foram considerados por PENG et al. (2007) como indicadores de bons ajustes para isotermas de sorção, para propósitos práticos, e serão tomados como referencia no presente trabalho.

$$
\mathrm{P}=\frac{100}{\mathrm{n}} \sum_{\mathrm{i}=1}^{\mathrm{n}} \frac{\left|\mathrm{m}_{\text {exp }}-\mathrm{m}_{\mathrm{pre}}\right|}{\mathrm{m}_{\exp }}
$$

onde: $\mathrm{m}_{\exp }$ e $\mathrm{m}_{\text {pre }}$ são umidades experimentais e preditas, respectivamente e $\mathrm{n}$ o número de observações.

\section{Determinação da monocamada}

A monocamada $\left(\mathrm{m}_{\mathrm{o}}\right)$ foi determinada através da equação de BET linearizada (Equação 2), com auxílio do aplicativo Microsoft Office Excel 2003 (2002).

$$
\frac{\mathrm{a}_{\mathrm{w}}}{\left(1-\mathrm{a}_{\mathrm{w}}\right) \cdot \mathrm{m}}=\frac{1}{\mathrm{~m}_{\mathrm{o}} \cdot \mathrm{C}}+\frac{(\mathrm{C}-1)}{\mathrm{m}_{\mathrm{o}} \cdot \mathrm{C}} \cdot \mathrm{a}_{\mathrm{w}}
$$

onde: $\mathrm{m}=$ umidade $\left(\mathrm{g} \mathrm{H}_{2} \mathrm{O} / 100\right.$ g b.s. $) ; \mathrm{a}_{\mathrm{w}}=$ atividade de água, $\mathrm{m}_{\mathrm{o}}=$ monocamada $\left(\mathrm{g}_{2} \mathrm{O} / 100 \mathrm{~g}\right.$ b.s.) e $\mathrm{C}=$ constante relacionada ao calor de sorção.

\section{Resultados e Discussão}

Isoterma de adsorção de umidade

A isoterma de adsorção (Figura 1) comportou-se como do Tipo II, de acordo com a classificação da IUPAC (1985), ou seja, apresenta forma de sigmóide. As isotermas do Tipo II são as mais frequentementes determinadas em alimentos, como por exemplo em fontes amiláceas (amidos de milho, batata, trigo e arroz), frutas (banana, ameixa), legumes (ervilhas secas, grãos de soja), sementes (algodão) e castanhas (castanha-de-caju, pistache) (Yanniots e Blahovec, 2009). Alimentos ricos em proteínas, como é o caso da castanha-do-Brasil (45,9\%) (Santos et al., 2010), 
também são conhecidos por atribuirem este formato à isoterma (Salwin, 1963), devido às ligações entre o substrato rico em proteínas e as moléculas de água.

Figura 1 - Isoterma de adsorção da castanha-do-Brasil a $25^{\circ} \mathrm{C}$.

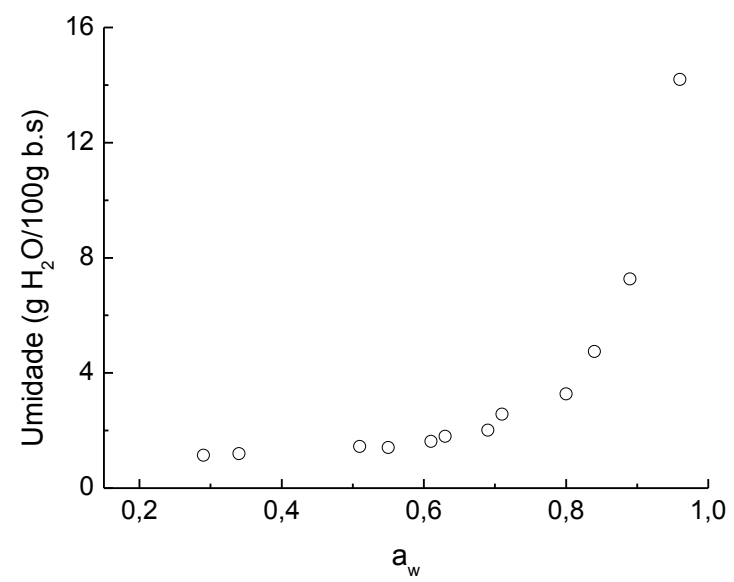

Durante a obtenção dos dados de adsorção (Tabela 2) não foram observadas, no produto, mudança de cor e aglomeração (perda de fluidez), em toda a faixa de umidade relativa estudada. No entanto, houve desenvolvimento de fungos em atividades de água superiores a 0,85. De acordo com os dados de adsorção, para que o produto apresente estabilidade microbiológica $\left(\mathrm{a}_{\mathrm{w}}<0,6\right)$, não deverá conter umidade superior a $1,62 \mathrm{~g} \mathrm{H}_{2} \mathrm{O} / 100 \mathrm{~g}$ b.s.

Tabela 2 - Dados de adsorção para a castanha-do-Brasil a $25^{\circ} \mathrm{C}$

\begin{tabular}{cc}
\hline $\mathbf{a}_{\mathbf{w}}$ & $\mathbf{m} \mathbf{( \mathbf { g ~ H }} \mathbf{\mathbf { O }} \mathbf{1 0 0 g} \mathbf{b . s .})$ \\
\hline 0,29 & 1,14 \\
0,34 & 1,19 \\
0,51 & 1,45 \\
0,55 & 1,41 \\
0,61 & 1,62 \\
0,63 & 1,80 \\
0,69 & 2,01 \\
0,71 & 2,57 \\
0,80 & 3,27 \\
0,89 & 4,75 \\
0,96 & 7,26 \\
\hline
\end{tabular}

$\mathrm{a}_{\mathrm{w}}=$ atividade de água; b.s. = base seca.

Dessa forma, a castanha-do-Brasil, quando armazenada em ambientes com elevada umidade relativas do ar, demandará maiores cuidados. Considerando a elevada umidade relativa do ar na região amazônica (67-90\%) (Bastos, 2005), e sendo esta região a maior produtora de castanha-doBrasil, recomenda-se a utilização de uma embalagem apropriada, ou seja, impermeável ao vapor de água, para garantir maior estabilidade dos constituintes intrínsecos do produto. 
$\mathrm{O}$ valor da monocamada, determinado pela equação de BET linearizada, foi de $0,7 \mathrm{~g} \mathrm{H}_{2} \mathrm{O} / 100 \mathrm{~g}$ em base seca, indicando a quase inexistência de constituintes com afinidade por moléculas de água no produto e, também, que não se deve secar o produto a níveis inferiores a $0,7 \mathrm{~g} \mathrm{H}_{2} \mathrm{O} / 100 \mathrm{~g}$ em base seca, evitando gasto desnecessário de energia. Ferreira e Pena (2003) encontraram valores de monocamada para farinha de pupunha (4,6\% de lipídios e $22,7 \%$ de proteínas) de 1,56 e 1,83g $\mathrm{H}_{2} \mathrm{O} / 100 \mathrm{~g}$ base seca para 15 e $35{ }^{\circ} \mathrm{C}$, respectivamente. Como nesse nível de umidade a $\mathrm{a}_{\mathrm{w}}$ do produto é inferior 0,2 , a estabilidade microbiológica da castanha-do-Brasil estará altamente assegurada.

\section{Modelagem matemática}

Na Tabela 3 encontram-se os valores dos coeficientes de determinação $\left(R^{2}\right)$ e desvios médios relativos $(\mathrm{P})$, obtidos a partir dos ajustes dos modelos matemáticos aos dados de adsorção da castanha-do-Brasil.

Tabela 3 - Coeficientes de determinação $\left(\mathrm{R}^{2}\right)$ e desvios médios relativos $(\mathrm{P})$ obtidos pelos ajustes.

\begin{tabular}{|c|c|c|c|}
\hline & Modelos & $\mathbf{R}^{2}$ & $\mathbf{P}$ \\
\hline \multirow{5}{*}{ 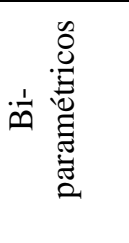 } & Kuhn & 0,9846 & 9,1 \\
\hline & Handerson & 0,9790 & 25,4 \\
\hline & Oswin & 0,9892 & 13,7 \\
\hline & Halsey & 0,9895 & 10,9 \\
\hline & Smith & 0,8791 & 45,7 \\
\hline \multirow{3}{*}{ 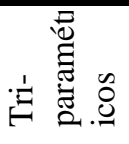 } & $\overline{\text { GAB }}$ & 0,9910 & 11,9 \\
\hline & BET & 0,9934 & 10,7 \\
\hline & BET modificada & 0,9910 & 11,9 \\
\hline
\end{tabular}

Analisando os valores de $\mathrm{R}^{2}$ e $\mathrm{P}$ constatou-se que os modelos bi-paramétricos de Kuhn e Halsey, e os tri-paramétricos de GAB, BET e BET modificada foram os que apresentaram os melhores ajustes na predição da isoterma de adsorção do produto, por exibirem os melhores coeficientes de determinação $\left(\mathrm{R}^{2} \approx 1\right)$ e os menores valores de $\mathrm{P}$. O modelo de GAB tem sido amplamente utilizado nos últimos anos, sendo aplicável para atividades de água até cerca de 0,9. Acima deste valor, o modelo de GAB normalmente prediz menor teor de água do que os observados experimentalmente (Yanniots e Blahovec, 2009).

As correlações entre os valores de umidade $(\mathrm{m})$, determinados a partir dos dados experimentais ( $\mathrm{m}$ observado) e preditos ( $\mathrm{m}$ predito) pelos modelos, para os modelos com os melhores ajustes (Figura 2), mostram excelente concordância entre os referidos valores, o que vem 
reforçar a aplicabilidade dos mesmos na predição da isoterma de adsorção da castanha-do-Brasil. Os modelos bi-paramétricos, por possuírem apenas dois parâmetros, apresentam mais fácil solução matemática; não necessitando de regressão não linear; sendo nesse caso os mais indicados; merecendo atenção especial o modelo de Kuhn, por apresentar o menor valor de P.

Figura 2 - Correlação entre m observado e m predito pelos diferentes modelos.

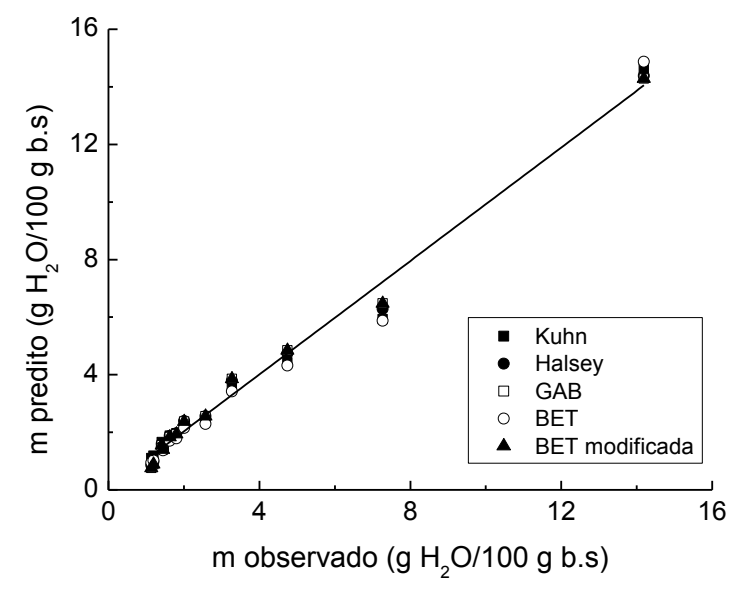

\section{Conclusão}

A isoterma de adsorção da castanha-do-Brasil comportou-se como do Tipo II, e para que apresente estabilidade microbiológica a umidade do produto não deve ser superior a 1,62 $\mathrm{g}$ $\mathrm{H}_{2} \mathrm{O} / 100 \mathrm{~g}$ b.s. A monocamada de $0,7 \mathrm{~g} \mathrm{H}_{2} \mathrm{O} / 100 \mathrm{~g}$ b.s. estabelece que o produto que não se deve seco a níveis inferiores a este, para evitar gasto desnecessário de energia. De acordo com os parâmetros de ajuste, as equações de Kuhn, Halsey, GAB, BET e BET modificada podem ser utilizadas na predição da isoterma de adsorção da castanha-do-Brasil. Em virtude do excelente ajuste da equação bi-paramétrica de Kuhn e por ser essa de mais fácil solução matemática, recomenda-se a sua utilização na predição da isoterma de adsorção da castanha-do-Brasil.

\section{Agradecimentos}

Ao CNPq (Conselho Nacional de Desenvolvimento Científico e Tecnológico) pelo suporte financeiro.

\section{Abstract}

The study of the hygroscopic behavior from Brazil-nut was realized building the isotherm of moisture adsorption at $25^{\circ} \mathrm{C}$, in the water activity (aw) range from 0.29 to 0.96. It was evaluated the applicability of eight mathematical models (Kuhn, Handerson, Oswin, Halsey, Smith, GAB, BET 
and modified-BET) in the prediction of adsorption isotherm. The product showed Type II isotherm. It was observed fungi development in aw exceeding 0.85. For microbiological stability $(a w<0.6)$ the product moisture must not exceed $1.62 \mathrm{~g} \mathrm{H}_{2} \mathrm{O} / 100 \mathrm{~g}$ b.s. The monolayer value was $0.7 \mathrm{~g}$ $\mathrm{H}_{2} \mathrm{O} / 100 \mathrm{~g}$ b.s., indicating almost absence of constituents with water affinity in the product. Kuhn, Halsey, GAB, BET e BET-modified equations were able to predict the product adsorption isotherm, and Kuhn equation is more suitable to be bi-parametric and having presented the best fit to the experimental points.

Key-words: Mathematical models, hygroscopicity, water activity.

\section{Referências}

ASSUNÇÃO, A. B.; PENA, R. S. Comportamento higroscópico do resíduo de camarão-rosa. Ciência e Tecnologia de Alimentos, n. 4, v. 27, p. 786-793, 2007.

BASTOS, T. X. Sistema de produção da pimenteira-do-reino. Sistemas de produção, 01. Embrapa Amazônia Oriental, 2005. Disponível online: http://sistemasdeproducao.cnptia.embrapa.br/FontesHTML/Pimenta/ PimenteiradoReino/paginas/clima.htm. Acesso em: 24/11/2010.

CHIRIFE, J.; IGLESIAS, H. A. Equations for fitting water sorption isotherms of foods: Part 1 - A review. International Journal of Food Science \& Technology, n. 3, v. 13, p. 159-174, 1978. http://dx.doi.org/10.1111/j.13652621.1978.tb00792.x

FERREIRA, C. D.; PENA, R. S.; Comportamento higroscópico da farinha de pupunha (Bactris gasipaes). Ciência e Tecnologia de Alimentos, n. 2, v. 23, p. 251-255, 2003. http://dx.doi.org/10.1590/S0101-20612003000200025

GLÓRIA, M. M.; REGITANO-D’ARCE, M. A. B. Concentrado e isolado protéico de torta de castanha-do-Pará: obtenção e caracterização química e funcional. Ciência e Tecnologia de Alimentos, n. 2, v. 20, p. 240-245, 2000.

IBGE. Instituto Brasileiro de Geografia e Estatística. Produção da extração vegetal e da silvicultura 1990-2009. Disponível em <http://www.sidra.ibge.gov.br/bda/pesquisas/pevs/default.asp?o=25\&i=P>, acesso em: 24/11/2010.

IUPAC - International Union of Pure and Applies Chemistry. Reporting physisorption data for gas/solid systems. Pure and Applied Chemistry, n. 4, v. 57, p. 603-619, 1985.

JAAFAR, F.; MICHALOWSKI, S. Modified BET equation for sorption/desorption isotherms. Drying Technology, n. 4, v. 8, p. 811-127, 1990. http://dx.doi.org/10.1080/07373939008959916

LABUZA, T. P.; Interpretation of sorption data in relation to the state of constituent water. In R. Duckworth (Ed.), Water relations in foods. New York: Academic Press, p. 155-172, 1975.

MAROULIS, Z. B.; TSAMI, E.; ARINOS-KOURIS, D.; SARAVACOS, G. D. Application of the GAB model to the sorption isotherms for dried fruits. Journal of Food Engineering, n. 1, v. 7, p. $63-70, \quad 1988$. http://dx.doi.org/10.1016/0260-8774(88)90069-6

MICROSOFT OFFICE EXCEL 2003. Microsoft Office Standard Edição 2003 para alunos e professors. Quito (Equador): SIGNUM, Cia. Ltda., 2002.

MYHARA, R. M.; SABLANI, S. S.; AL-ALAWI, S. M.; TAYLOR, M. S.; Water sorption isotherms of dates: modeling using GAB equation and artificial neural network approaches. Lebensmittel-Wissenschaft undTechnologie, n. 7-8, v. 31, p. 699-706, 1998.

PARK, K. J.; NOGUEIRA, R. I. Modelos para ajuste de isotermas de sorção de alimentos. Engenharia Rural, n. 1, v. 3, p. 80-6, 1992.

PENG, G.; CHEN, X.; WU, W.; JIANG, X. Modeling of water sorption isotherm for corn starch. Journal of Food Engineering, n. 2, v. 80, p. 562-567, 2007. http://dx.doi.org/10.1016/j.jfoodeng.2006.04.063

SALWIN, H. Moisture levels required for stability in dehydrated foods. Food Technology, n. 9, v. 17, p. 1114-1121, 1963. 
SANTOS, O. V.; LOPES, A. S.; AZEVEDO, G. O.; SANTOS, A. C. Processing of Brazil-nut flour: characterization, thermal and morphological analysis. Ciência e Tecnologia de Alimentos, n. 1, v. 30, p. 264-269, 2010. http://dx.doi.org/10.1590/S0101-20612010000500040

SOUZA, M. L.; MENEZES, H. C. Processamento de amêndoa e torta de castanha-do-Brasil e farinha de mandioca: parâmetros de qualidade. Ciência e Tecnologia de Alimentos, n. 1, v. 24, p. 120-128, 2004. http://dx.doi.org/10.1590/S0101-20612004000100022

STATISTICA for Windows 5.5. Computer Program Manual. Tulsa: StatSoft, Inc., 2000.

YANNIOTS, S.; BLAHOVEC, J. Model analysis of sorption isotherms. LWT - Food Science and Technology, v. 42 , n. 10, p. 1688-1695, 2009.

Submetido em 26 nov 2010, Aceito para publicação em 21 mai. 2012. 